\title{
Comparative Study Among Lease Square Method, Steepest Descent Method, and Conjugate Gradient Method for Atmopsheric Sounder Data Analysis
}

\author{
Kohei Arai ${ }^{1}$ \\ Graduate School of Science and Engineering \\ Saga University \\ Saga City, Japan
}

\begin{abstract}
Comparative study among Least Square Method: LSM, Steepest Descent Method: SDM, and Conjugate Gradient Method: CGM for atmospheric sounder data analysis (estimation of vertical profiles for water vapor) is conducted. Through simulation studies, it is found that CGM shows the best estimation accuracy followed by SDM and LSM. Method dependency on atmospheric models is also clarified.
\end{abstract}

Keywords-nonlinear optimization theory; solution space; atmpspheric sounder

\section{INTRODUCTION}

Air-temperature and water vapor profiles are used to be estimated with Infrared Sounder data [1]. One of the problems on retrieving vertical profiles is its retrieving accuracy. In particular, estimation accuracy of air-temperature and water vapor at tropopause ${ }^{1}$ altitude is not good enough because there are gradient changes of air-temperature and water vapor profile in the tropopause so that observed radiance at the specific channels are not changed for the altitude.

In order to estimate air-temperature and water vapor, least square based method is typically used. In the process, Root Mean Square: RMS difference between observed radiance and calculated radiance with the designated physical parameters are minimized. Then the designated physical parameters including air-temperature and water vapor at the minimum RMS difference are solutions.

Typically, Newton-Raphson method ${ }^{2}$ which gives one of local minima is used for minimization of RMS difference. Newton-Raphson needs first and second order derivatives, Jacobean and Hessian at around the current solution. It is not easy to formularize these derivatives analytically. The proposed method is based on Levenberg Marquardt: LM of non-linear least square method ${ }^{3}$. It uses numerically calculated first and second order derivatives instead of analytical based derivatives. Namely, these derivatives can be calculated with radiative transfer model based radiance calculations. At

${ }^{1}$ http://en.wikipedia.org/wiki/Tropopause

${ }^{2}$ http://en.wikipedia.org/wiki/Newton's_method

http://en.wikipedia.org/wiki/Levenberg\%E2\%80\%93Marquardt_algorithm around the current solution in the solution space, directional derivatives are calculated with the radiative transfer model.

The proposed method is validated for air-temperature and water vapor profile retrievals with Infrared: IR sounder ${ }^{4}$ data derived from Atmospheric Infrared Sounder:/AIRS onboard AQUA satellite [2]-[7]. A comparison of retrieving accuracy between Newton-Raphson method and the proposed method based on LM method [8] is made in order to demonstrate an effectiveness of the proposed method in terms of estimation accuracy in particular for the altitude of tropopause [9]. Global Data Assimilation System: GDAS ${ }^{5}$ data of assimilation model derived 1 degree mesh data is used as truth data of airtemperature and water vapor profiles. The experimental data show that the proposed method is superior to the conventional Newton-Raphson method.

The following section describes proposed method for water vapor profile retrievals followed by experiments. Then finally, conclusion and some discussions are described.

\section{THEORETICAL BACKGROUND AND SIMULATION METHOD}

\section{A. Radiative Transfer Equation}

Radiative transfer equation is expressed with equation (1).

$R v=\left(I_{0}\right)_{v} \tau_{v}\left(z_{0}\right)+\int_{z_{0}}^{\infty} B v\{T(z)\} K_{v}(z) d z$

where $v$ denotes wave number $(\mathrm{cm}-1)$, and

$R_{v}$ : at sensor brightness temperature

$\left(I_{0}\right)_{v}$ : brightness temperature of ground surface

$\tau_{v}\left(z_{0}\right)$ : total column atmospheric transmittance

$B\{T(z)\} v$ : Planckian function of air temperature at the altitude

of $\mathrm{z}$

$K_{v}(z)$ : atmospheric transmittance at the altitude of $\mathrm{z}$

This equation (1) can be linearized as follows,

$R=B K$

${ }^{4}$ http://en.wikipedia.org/wiki/Atmospheric_Infrared_Sounder

${ }^{5} \mathrm{http}: / /$ www.mmm.ucar.edu/mm5/mm5v3/data/gdas.html 
Where the number of unknown variables and the number of given equations are same. Therefore, it can be solved relatively easily. This solution from linear inversion provides initial value of the steepest descent method. Without this initial value, steepest descent method falls in one of local minima easily.

\section{B. Water Vapor Profile Retrieval Method}

For instance, it can be solved based on steepest descent method as shown in equation (3)

$$
R-R_{0}=\frac{\partial R}{\partial q}\left(q-q_{0}\right)
$$

Also, it is possible to estimate water vapor profile to minimize the following covariance matrix of error,

$$
\hat{x}=x_{a}+\left(A^{T} S_{\varepsilon}^{-1} A+S_{a}^{-1}\right)^{-1} A^{T} S_{\varepsilon}^{-1}\left(R-R_{a}\right)
$$

Where

$x_{a}$ : Designated variable matrix

$x^{\wedge}$ : Variable matrix for estimation

A: Jacobian Matrix

$S$ : Covariance matrix for measurement error

$R$ : Observed brightness temperature

$R_{a}$ : Estimated brightness temperature

Covariance matrix can be defined as equation (5).

$$
S_{i j}=\varepsilon\left(x_{i}-\hat{x}_{i}\right)\left(x_{j}-\hat{x}_{j}\right)^{T}
$$

Jacobian Matrix can be expressed in equation (6).

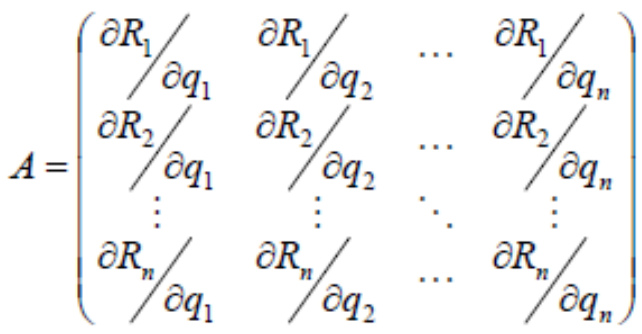

\section{Steepest Descent Method (Non-linear optimization method)}

Steepest descent method can be represented in equation (7).

$$
q_{k}=q_{k-1}+\alpha_{k} g_{k}
$$

Where

$q_{k}$ : estimated value at the iteration number $\mathrm{k}$

$g:$ updating vector

$\alpha:$ step width

Estimated value can be updated with the direction of $g$ and with step size of $\alpha$. Then estimation process is converged at one of local minima, not global optimum solution. This learning or updating process can be illustrated as shown in Figure 1. Initial value is derived from the linear inversion, $K=B^{-1} R$.

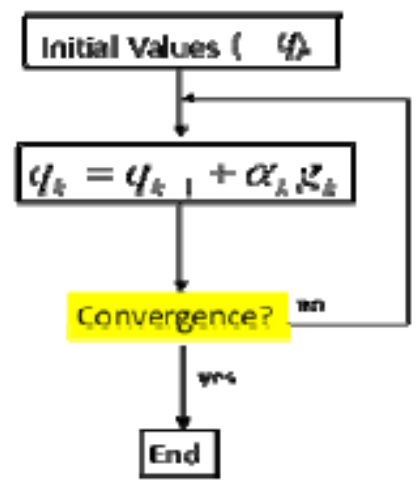

Fig. 1. Process flow of steepest descent method

\section{Simulation Method}

From equation (1), observed brightness temperature of atmospheric sounder can be expressed as follows,

$$
R_{v}=\alpha+\beta e^{-\left(\omega_{w}+\phi\right)}
$$

Where $w$ denotes water vapor content in the atmosphere while $\alpha 、 \beta 、 \gamma 、 \psi$ denotes coefficients. Using MODTRAN of radiative transfer software code including six atmospheric models, Tropic: TRP, Mid. Latitude Summer: MLS, Mid. Latitude Winter: MLW, Sub-Arctic Summer: SAS, Sub-Arctic Winter: SAW, and 1976 US Standard atmosphere: USS, observed brightness temperature at certain wavelength can be calculated. With the reference to AIRS observation wavelength, the following three wavelength are selected for simulation study, 6.7, 7.3, and $7.5 \mu \mathrm{m}$. Therefore, coefficients in equation (8) can be estimated for each observation wavelength together with Root Mean Square Error: RMSE of water vapor retrieval error.

\section{Simulation RESUlts}

\section{A. Water Vapor Profile}

Figure 2 to 8 shows water vapor profiles for 6 different atmospheric models with default relative humidity, and its plus minus $10 \%, 20 \%$, and $30 \%$ while Figure 9 to 15 shows accumulated water vapor profiles for 6 different atmospheric models with default relative humidity, and its plus minus $10 \%$, $20 \%$, and $30 \%$ derived from MODTRAN 4.3, respectively. These water vapor profiles and accumulated water vapor profiles are totally dependent on relative humidity, obviously. It is also obvious that water vapor and accumulated water vapor of the Tropic atmosphere is greatest followed by Mid. Latitude Summer, 1976 U.S. Standard, Mid. Latitude Winter, Sub Arctic Summer, and Sub Arctic Winter. 


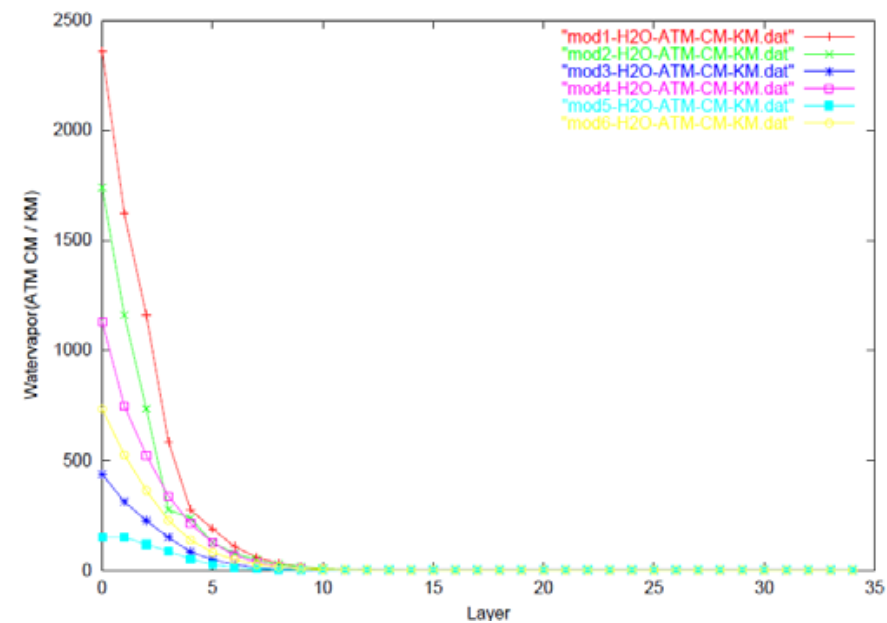

Fig. 2. Water vapor profiles for 6 atmospheric models with default relative Humidity

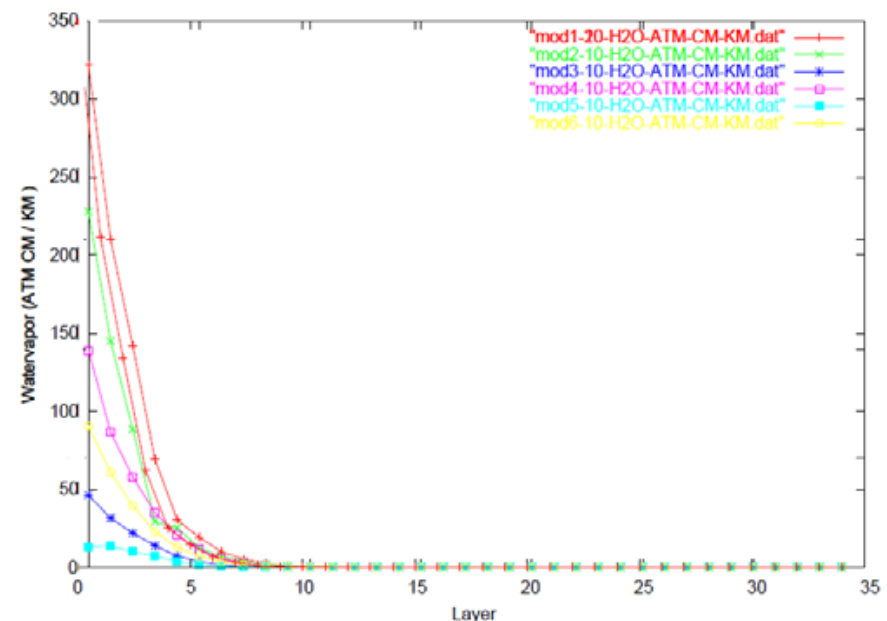

Fig. 3. Water vapor profiles for 6 atmospheric models with default relative humidity minus $10 \%$

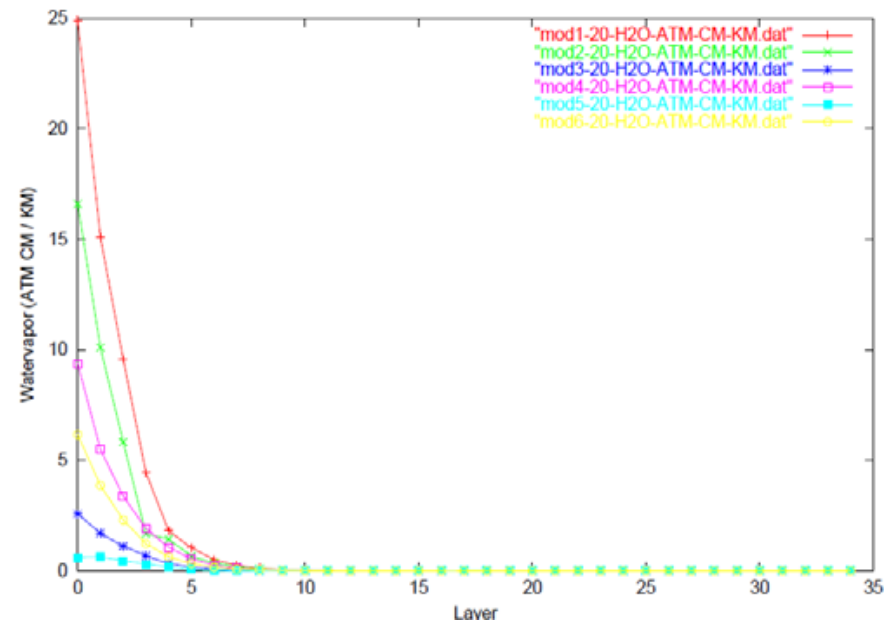

Fig. 4. Water vapor profiles for 6 atmospheric models with default relative humidity minus $20 \%$

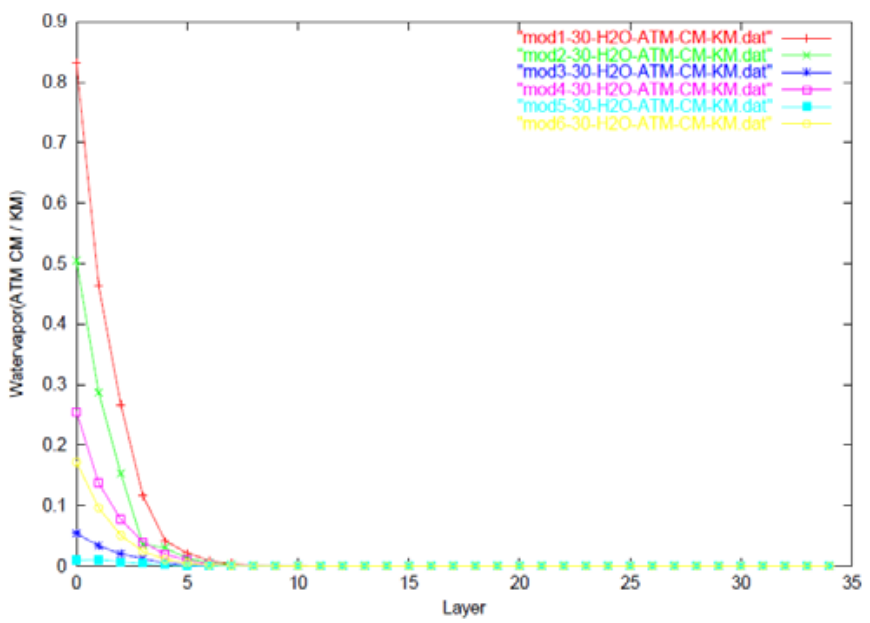

Fig. 5. Water vapor profiles for 6 atmospheric models with default relative humidity minus $30 \%$

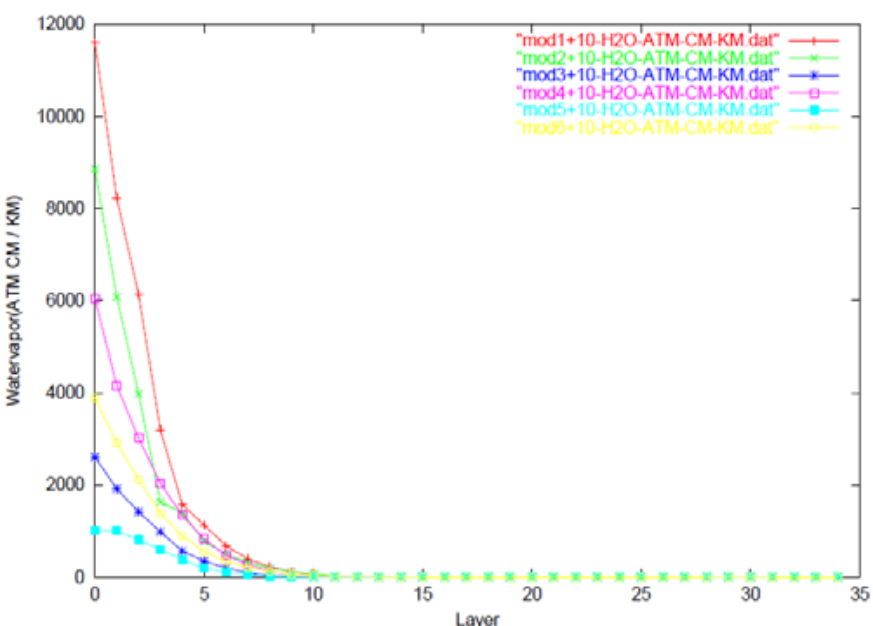

Fig. 6. Water vapor profiles for 6 atmospheric models with default relative humidity plus $10 \%$

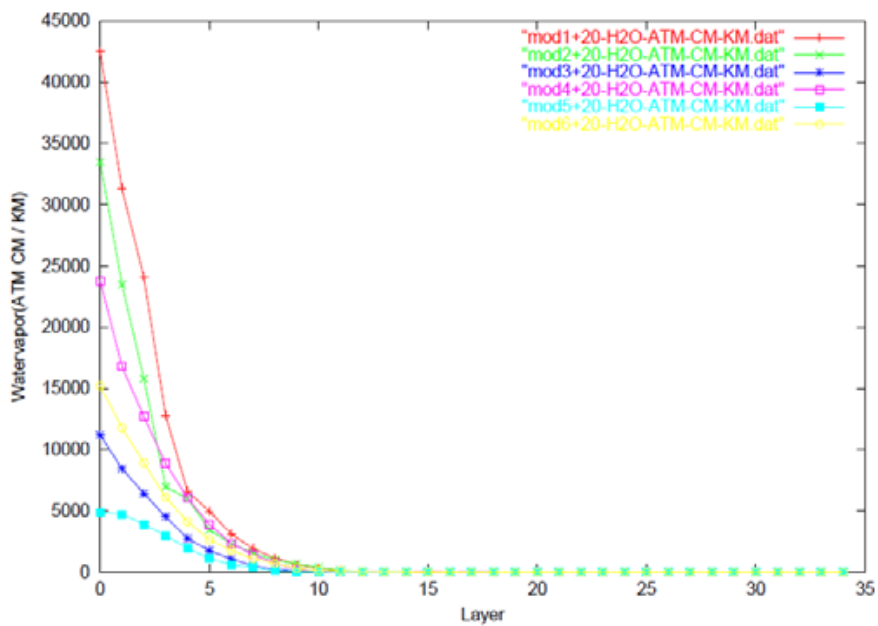

Fig. 7. Water vapor profiles for 6 atmospheric models with default relative humidity plus $20 \%$ 


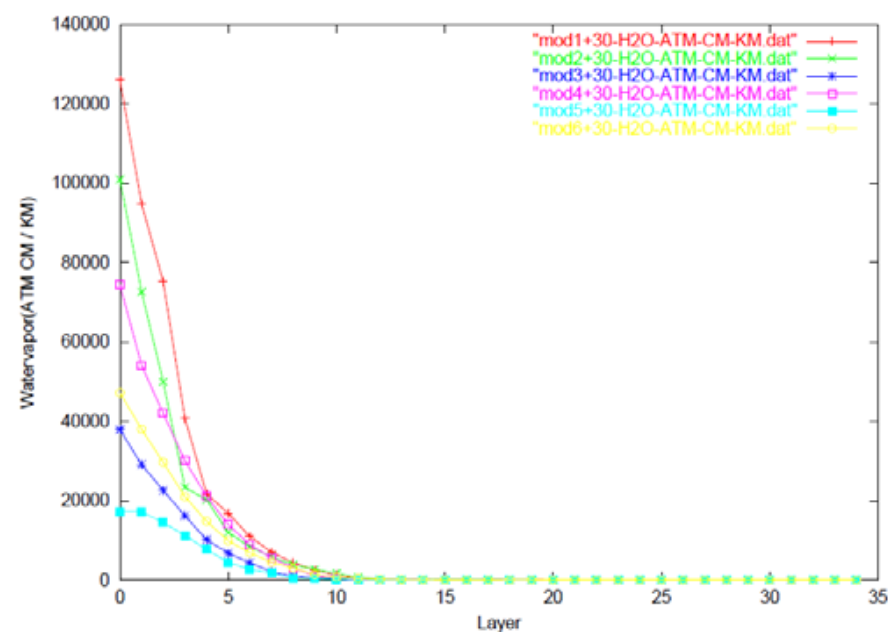

Fig. 8. Water vapor profiles for 6 atmospheric models with default relative humidity plus $30 \%$

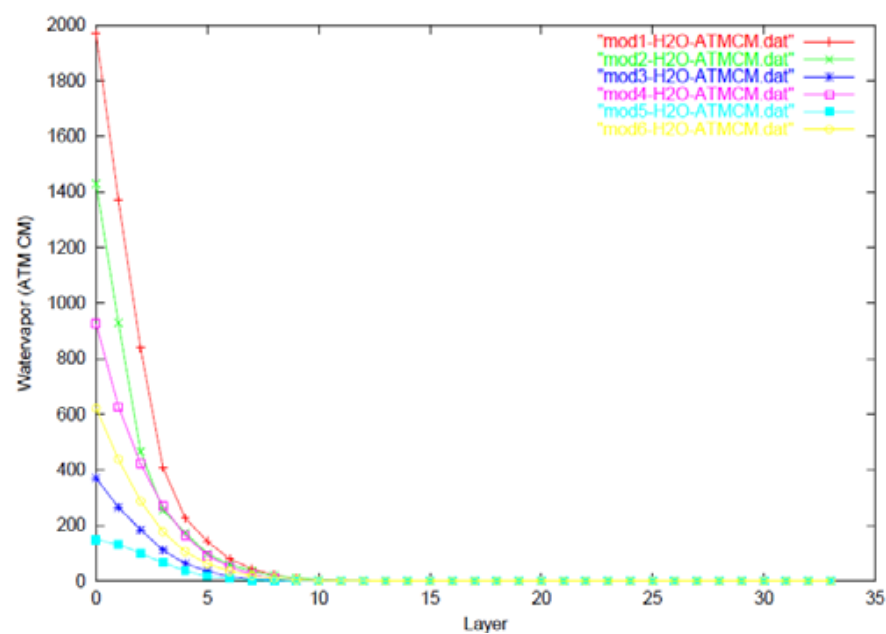

Fig. 9. Accumulative water vapor profiles for 6 atmospheric models with default relative humidity

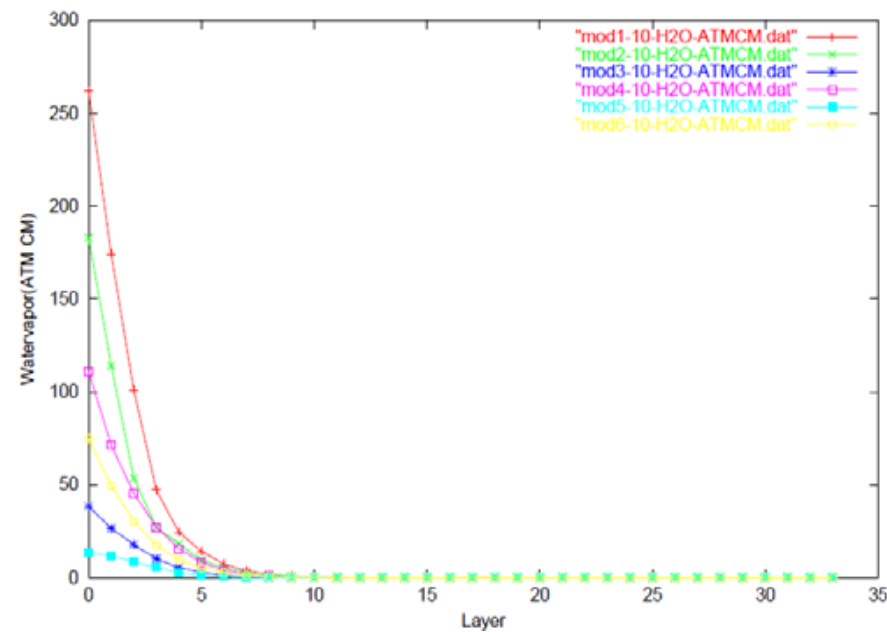

Fig. 10. Accumulative water vapor profiles for 6 atmospheric models with default relative humidity minus $10 \%$

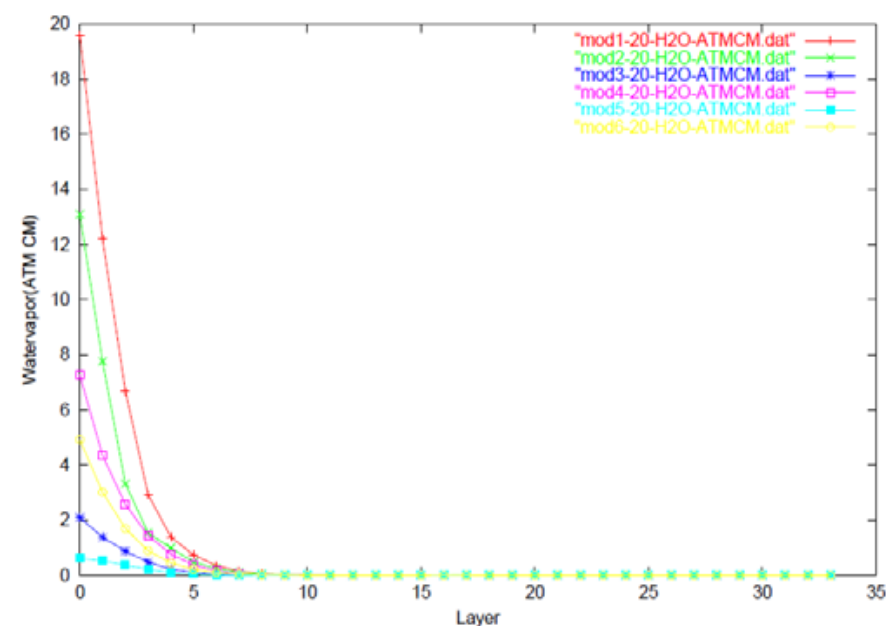

Fig. 11. Accumulative water vapor profiles for 6 atmospheric models with default relative humidity minus $20 \%$

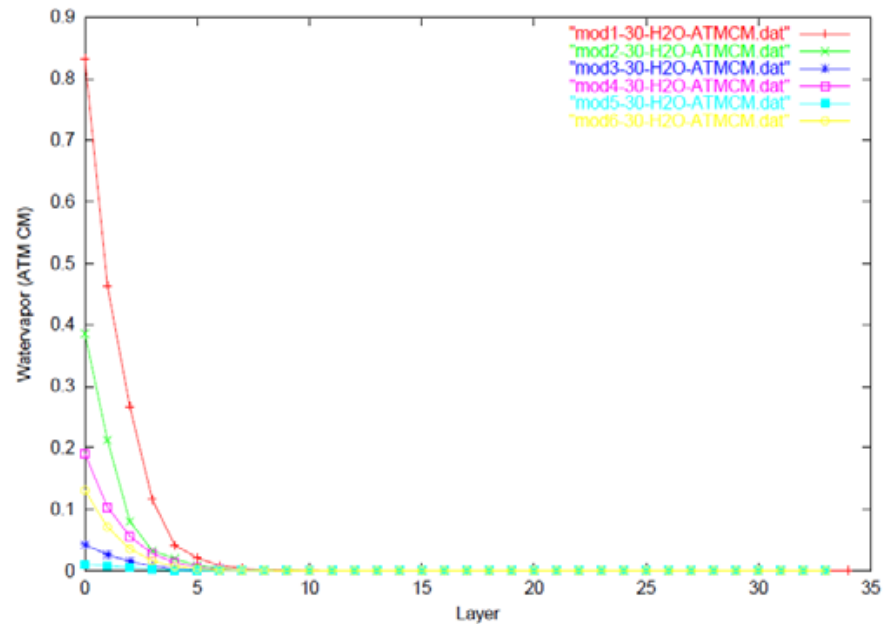

Fig. 12. Accumulative water vapor profiles for 6 atmospheric models with default relative humidity minus $30 \%$

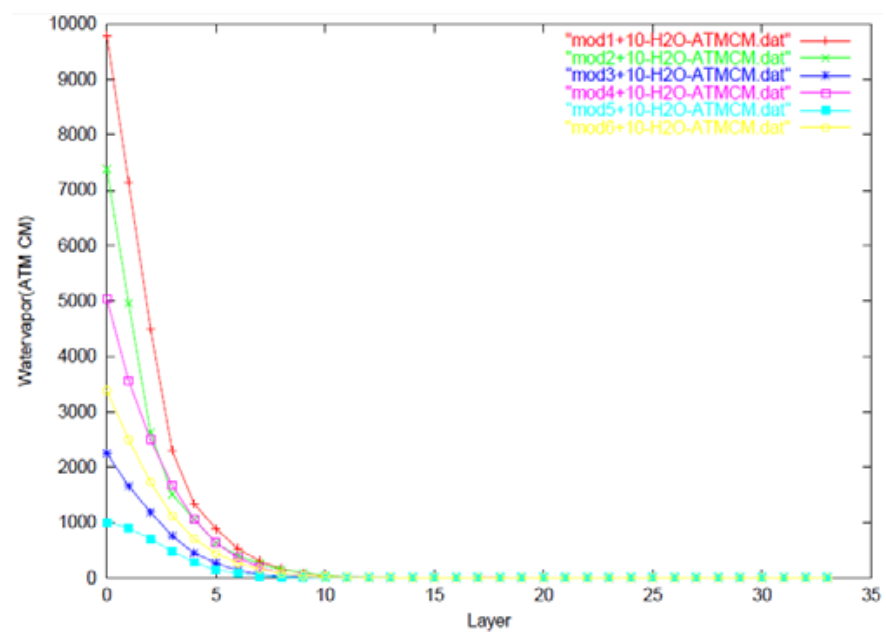

Fig. 13. Accumulative water vapor profiles for 6 atmospheric models with default relative humidity plus $10 \%$ 


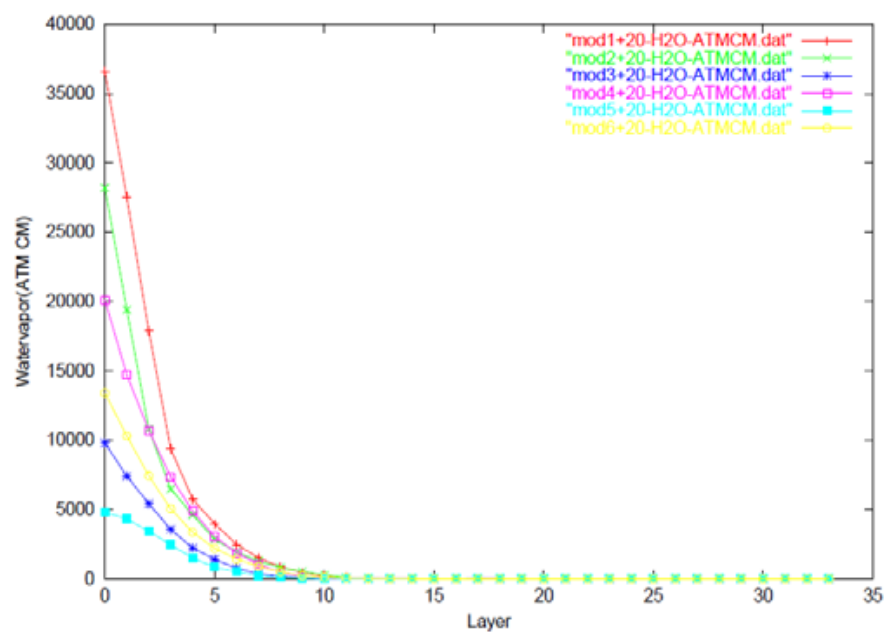

Fig. 14. Accumulative water vapor profiles for 6 atmospheric models with default relative humidity plus $20 \%$

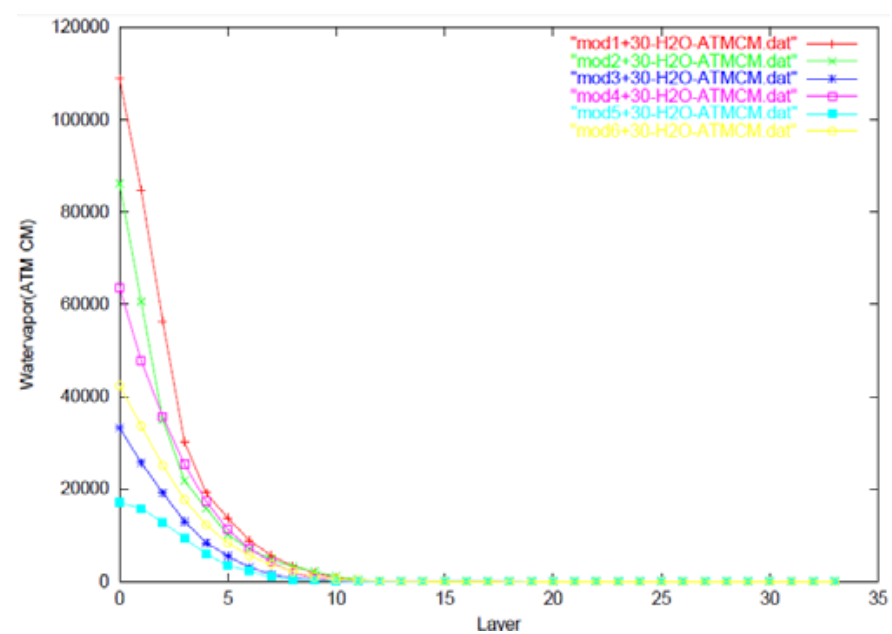

Fig. 15. Accumulative water vapor profiles for 6 atmospheric models with default relative humidity plus $30 \%$

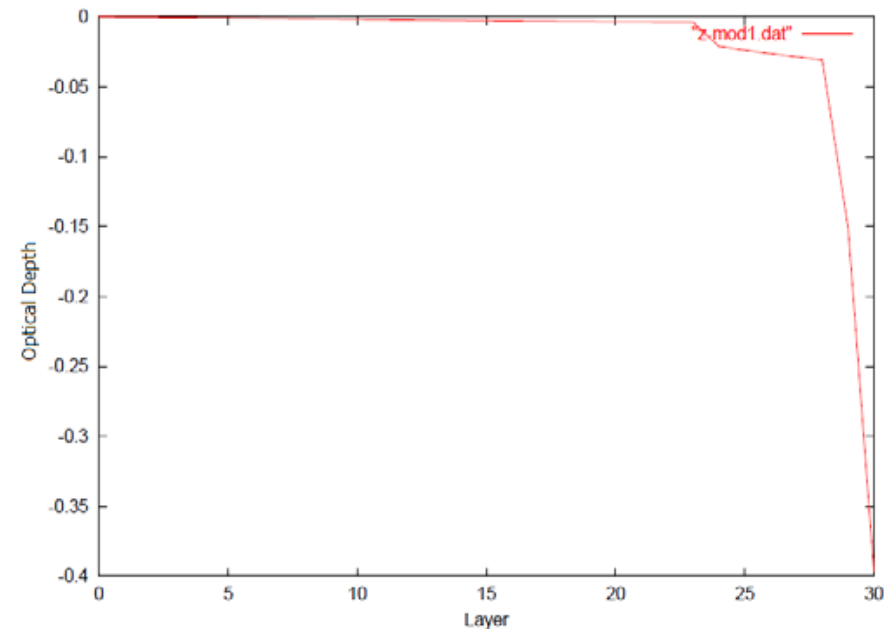

Fig. 16. Optical depth profile for the tropic atmospheric model

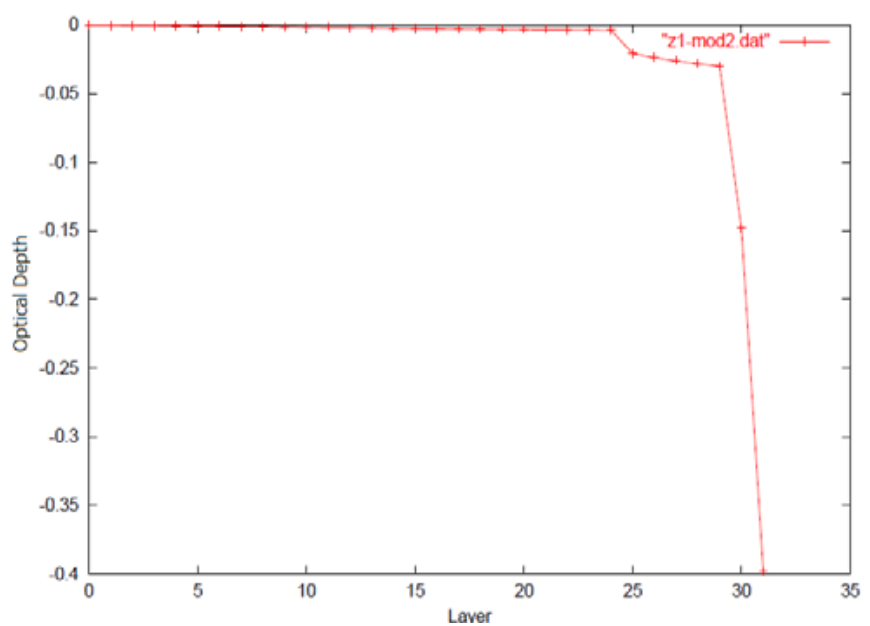

Fig. 17. Optical depth profile for the Mid. Latitude Summer atmospheric Model

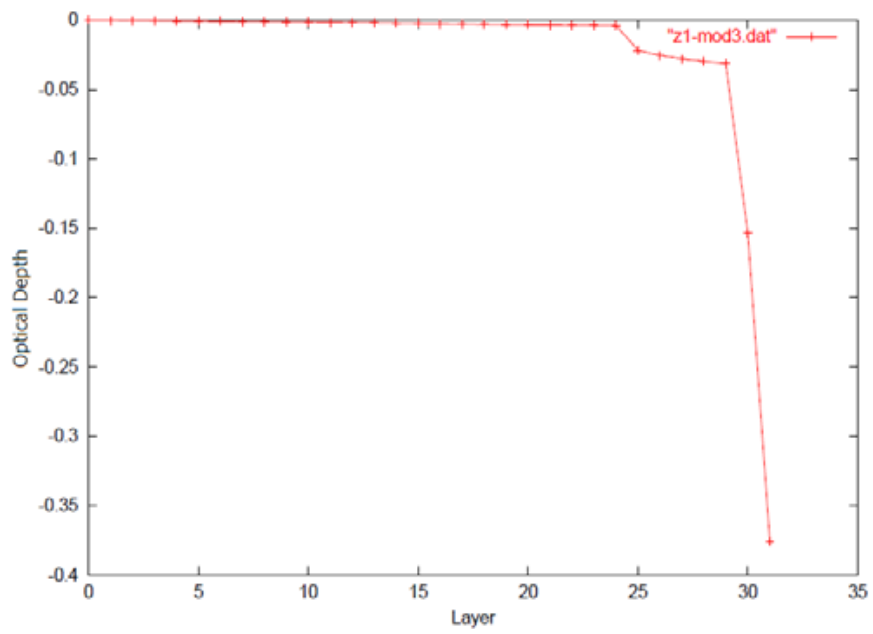

Fig. 18. Optical depth profile for the Mid. Latitude Winter atmospheric model

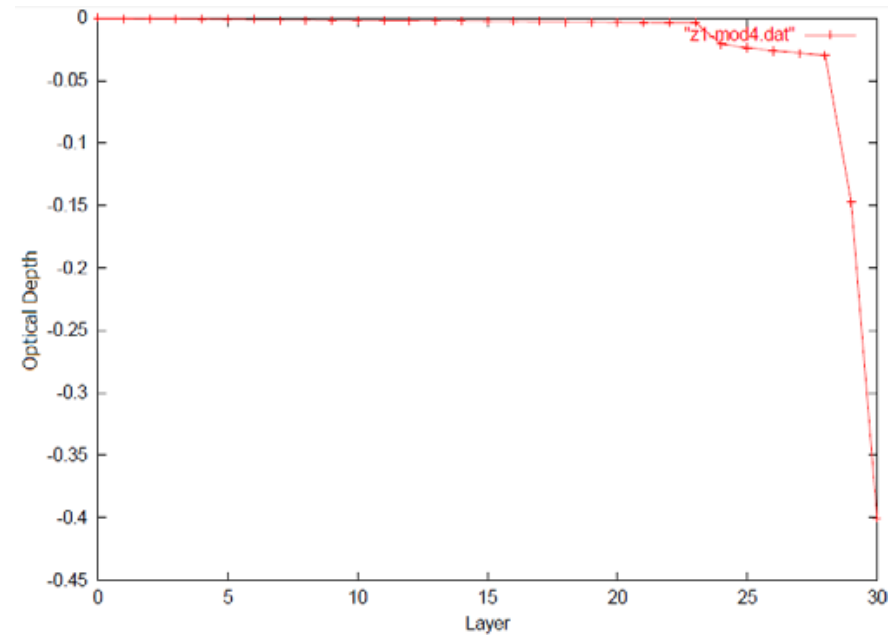

Fig. 19. Optical depth profile for the Sub Arctic Summer atmospheric model 


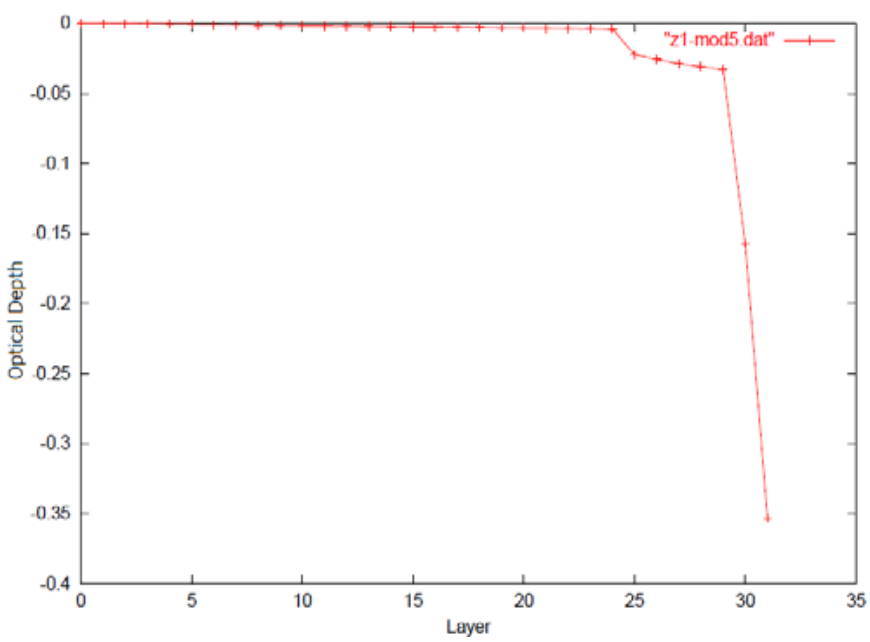

Fig. 20. Optical depth profile for the Sub Arctic Winter atmospheric model

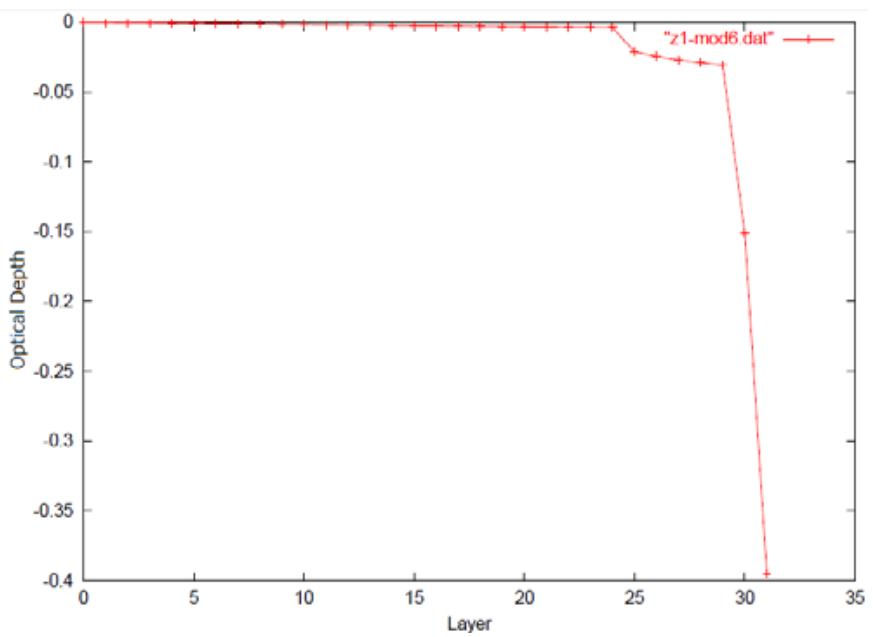

Fig. 21. Optical depth profile for the 1976 U.S. Standard atmospheric model

\section{B. Optical Depth Profile}

Figure 16 to 21 shows optical depth profiles for 6 different atmospheric models. It is also obvious that optical depth of the Tropic atmosphere is greatest followed by Mid. Latitude Summer, 1976 U.S. Standard, Mid. Latitude Winter, Sub Arctic Summer, and Sub Arctic Winter.

\section{Up-welling Radiance Profile}

Figure 22 to 26 shows up-welling radiance profiles for 6 different atmospheric models. It is also obvious that upwelling radiance of the Tropic atmosphere is greatest followed by Mid. Latitude Summer, 1976 U.S. Standard, Mid. Latitude Winter, Sub Arctic Summer, and Sub Arctic Winter.

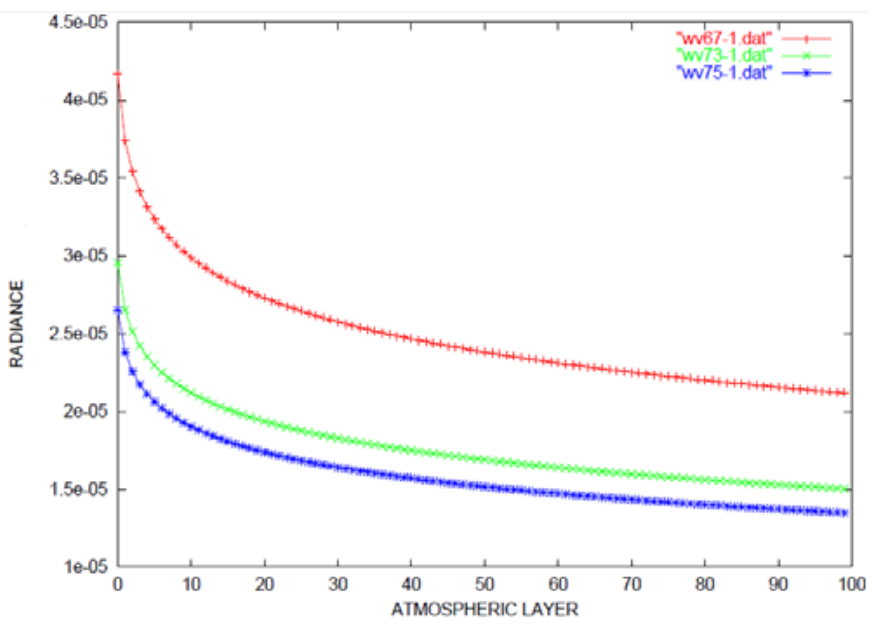

Fig. 22. Up-welling radiance profiles for the Tropic atmospheric model

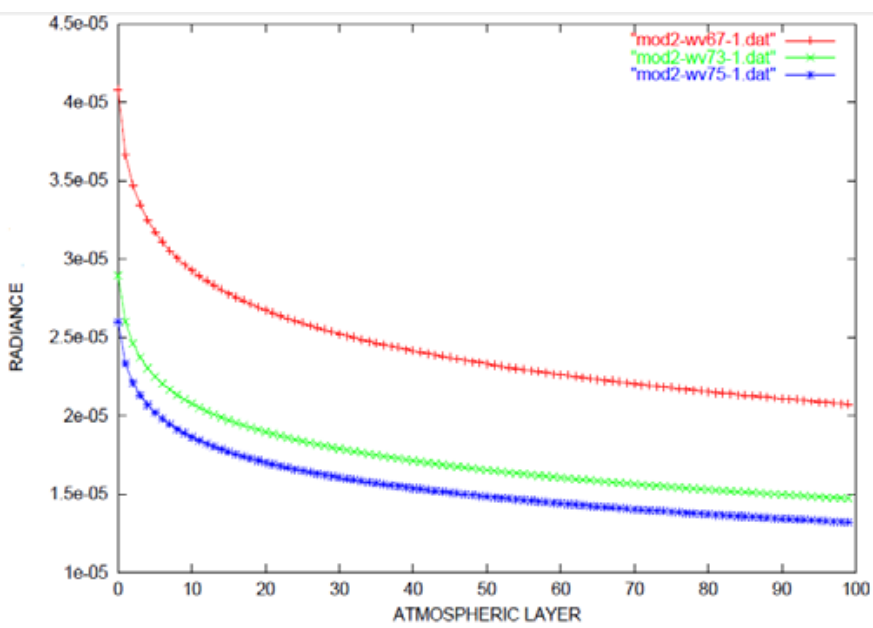

Fig. 23. Up-welling radiance profiles for the Mid. Latitude Summer atmospheric model

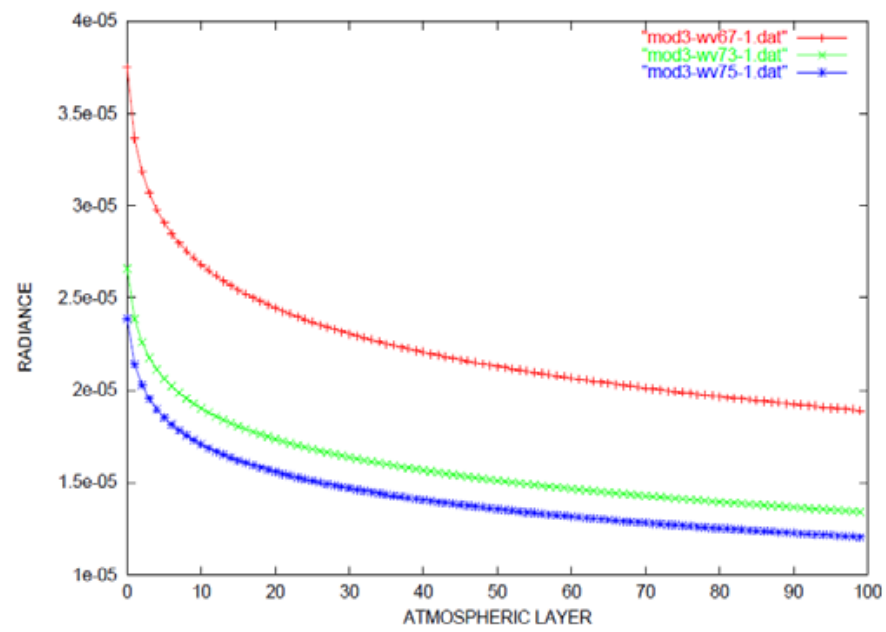

Fig. 24. Up-welling radiance profiles for the Mid. Latitude Winter atmospheric model 


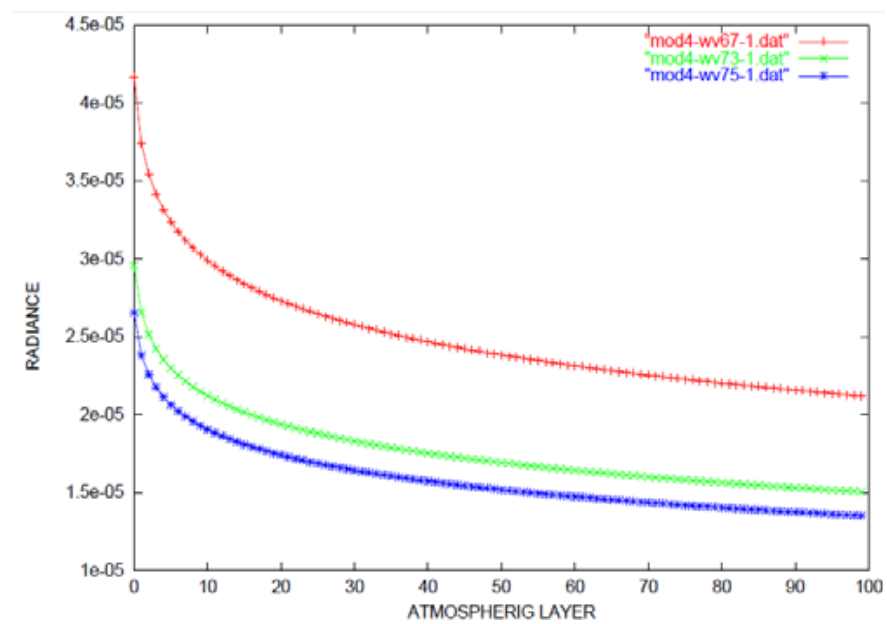

Fig. 25. Up-welling radiance profiles for the Sub Arctic Summer atmospheric model

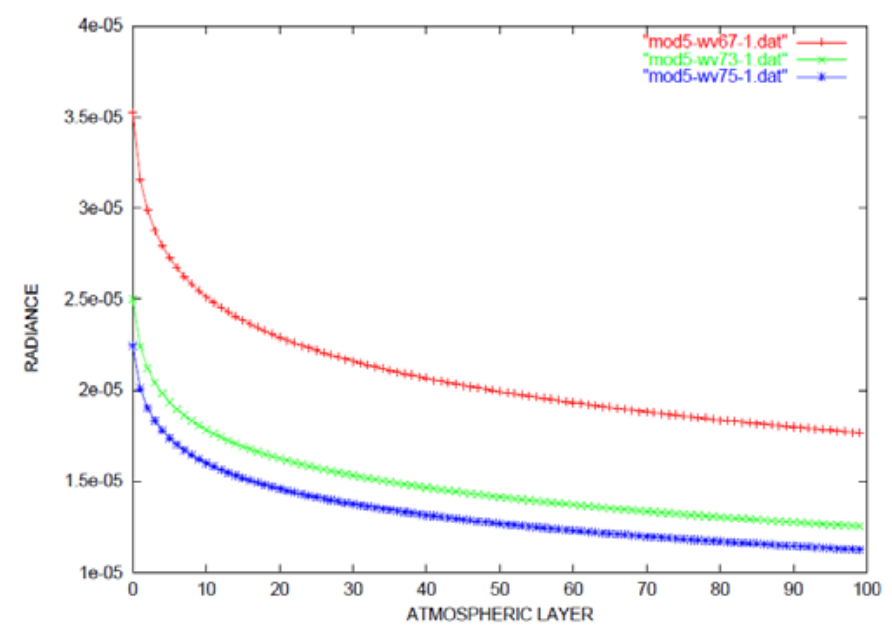

Fig. 26. Up-welling radiance profiles for the Sub Arctic Winter atmospheric Model

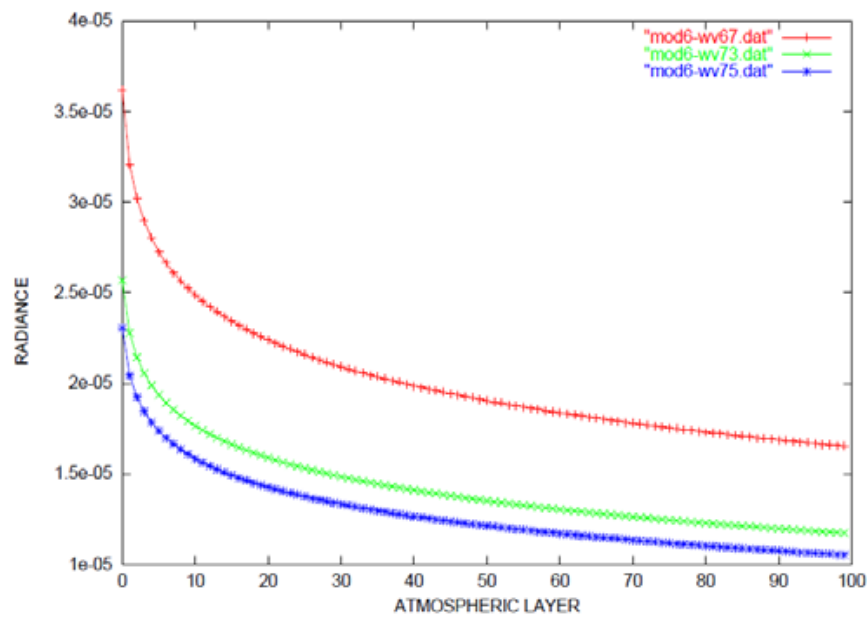

Fig. 27. Up-welling radiance profiles for the 1976 U.S. Standard atmospheric model

\section{RMSE for Three Different Methods for Water Vapor Profile Estimation}

Root Mean Square Error: RMSE of three different water vapor estimation methods are evaluated. Using spectral upwelling radiance, it is possible to estimate water vapor profile. With the reference to AIRS observation wavelength, the following three wavelengths are selected for simulation study, 6.7, 7.3, and $7.5 \mu \mathrm{m}$. Up-welling radiance at the wavelength is calculated for 6 different atmospheric models with MODTRAN 4.3, then water vapor profile is estimated with the Least Square Method, Steepest Descent Method, and Conjugate Gradient Method. True water vapor profiles are given by MODTRAN 4.3. Therefore, RMSE can be evaluated.

Table 1 to 3 shows RMSE. It is found that RMSE of the Conjugate Gradient Method is smallest followed by Steepest Descent Method and Least Square Method. Least Square Method is totally equal to Linear Regression. Because water vapor profile estimation is not linear problem solving, RMSE of the Least Square Method is not so good. Meanwhile, both Conjugate Gradient and Steepest Descent Methods find one of local minima. Steepest Descent Method often output trivial solution due to algorithm nature. Therefore, Conjugate Gradient Method is better than Steepest Descent Method mostly.

TABLE I. RMSE For SteEPEST Descent Method For 6 ATMOSPHERIC MODELS

\begin{tabular}{|c|c|c|c|}
\hline$\lambda(\mu m)$ & 6.7 & 7.3 & 7.5 \\
\hline$\alpha$ & $-9.9042927 \mathrm{e}-01$ & $-9.8428169 \mathrm{e}-01$ & $-9.8428161 \mathrm{e}-01$ \\
\hline$\beta$ & $-5.804411 \mathrm{e}-01$ & $-1.2968778 \mathrm{e}+00$ & $-2.0132363 \mathrm{e}+00$ \\
\hline$\gamma$ & $6.1027836 \mathrm{e}-01$ & $1.2968778 \mathrm{e}+00$ & $2.0132363 \mathrm{e}+00$ \\
\hline$\psi$ & $2.1518239 \mathrm{e}-02$ & $2.3999963 \mathrm{e}-02$ & $2.3999963 \mathrm{e}-02$ \\
\hline RMSE $($ atom $\cdot \mathrm{cm})$ & $1.286716 \mathrm{e}-02$ & $1.283178 \mathrm{e}-02$ & $1.283175 \mathrm{e}-02$ \\
\hline
\end{tabular}

TABLE II. RMSE For CONJUGATE GRADIENT METHOD For 6 ATMOSPHERIC MODELS

\begin{tabular}{|c|c|c|c|}
\hline$\lambda(\mu m)$ & 6.7 & 7.3 & 7.5 \\
\hline$\alpha$ & $-9.9469977 \mathrm{e}-01$ & $-1.0010890 \mathrm{e}+00$ & $-9.9546792 \mathrm{e}-01$ \\
\hline$\beta$ & $1.0482258 \mathrm{e}-01$ & $-4.8530780 \mathrm{e}-02$ & $3.9825363 \mathrm{e}-02$ \\
\hline$\gamma$ & $2.2675326 \mathrm{e}-01$ & $3.0309994 \mathrm{e}-01$ & $2.7884224 \mathrm{e}-01$ \\
\hline$\psi$ & $2.2841551 \mathrm{e}-01$ & $1.2047212 \mathrm{e}-01$ & $3.2866798 \mathrm{e}-02$ \\
\hline RMSE $($ atom $\cdot c m)$ & $3.711227 \mathrm{e}-03$ & $3.818651 \mathrm{e}-03$ & $3.711227 \mathrm{e}-03$ \\
\hline
\end{tabular}

TABLE III. RMSE For LEAST SQUARE METHOd For 6 ATMOSPHERIC MODELS

\begin{tabular}{|c|c|c|c|}
\hline$\lambda(\mu \mathrm{m})$ & 6.7 & 7.3 & 7.5 \\
\hline$\alpha$ & $-4.6402598 \mathrm{e}+00$ & $-1.4154191 \mathrm{e}+01$ & $-1.4366376 \mathrm{e}+01$ \\
\hline$\beta$ & $1.3551852 \mathrm{e}+00$ & $-9.7287497 \mathrm{e}-05$ & $1.0303786 \mathrm{e}-01$ \\
\hline$\gamma$ & $-3.1595048 \mathrm{e}-04$ & $2.4652031 \mathrm{e}+00$ & $-1.9849016 \mathrm{e}-03$ \\
\hline$\psi$ & $7.4871903 \mathrm{e}+00$ & $-2.6431300 \mathrm{e}+03$ & $1.5466422 \mathrm{e}+00$ \\
\hline RMSE $($ atom $\cdot \mathrm{cm})$ & $1.946242 \mathrm{e}+01$ & $8.258933 \mathrm{e}-01$ & $7.951221 \mathrm{e}-01$ \\
\hline
\end{tabular}




\section{CONCLUSION}

Comparative study among Least Square Method: LSM, Steepest Descent Method: SDM and Conjugate Gradient Method: CGM for atmospheric sounder data analysis (estimation of vertical profiles for water vapor) is conducted. Three retrieval methods, SDM, LSM, and CGM are compared in terms of Root Mean Square Error: RMSE. In particular, atmospheric model dependency on RMSE is to be clarified. Thus it becomes possible to use the most appropriate method for each atmospheric model. Through simulation studies, it is found that CGM shows the best estimation accuracy followed by SDM and LSM. Method dependency on atmospheric models is also clarified.

\section{ACKNOWLEDGMENT}

The author would like to thank Mr. Chen Ding for his effort to conduct simulation studies.

\section{REFERENCES}

[1] Kohei Arai, Lecture Note on Remote Sensing, Morikita-Shuppan publishing Co. Ltd, 2004.

[2] NASA/JPL, "AIRS Overview". NASA/JPL.http://airs.jpl.nasa.gov/overview/overview/.

[3] NASA "Aqua and the A-Train".

NASA.http://www.nasa.gov/mission_pages/aqua/.
[4] NASA/GSFC "NASA Goddard Earth Sciences Data and Information Services Center". NASA/GSFC.http://disc.gsfc.nasa.gov/AIRS/data_products.shtml.

[5] NASA/JPL "How AIRS Works". NASA/JPL.http://airs.jpl.nasa.gov/technology/how_AIRS_works.

[6] NASA/JPL "NASA/NOAA Announce Major Weather Forecasting Advancement". NASA/JPL.http://jpl.nasa.gov/news/news.cfm?release=2005-137.

[7] NASA/JPL "New NASA AIRS Data to Aid Weather, Climate Research". NASA/JPL. http://www.jpl.nasa.gov/news/features.cfm?feature=1424.

\section{AUTHORS PROFILE}

Kohei Aarai He received BS, MS and PhD degrees in 1972, 1974 and 1982, respectively. He was with The Institute for Industrial Science and Technology of the University of Tokyo from April 1974 to December 1978 and also was with National Space Development Agency of Japan from January, 1979 to March, 1990. During from 1985 to 1987, he was with Canada Centre for Remote Sensing as a Post Doctoral Fellow of National Science and Engineering Research Council of Canada. He moved to Saga University as a Professor in Department of Information Science on April 1990. He was a councilor for the Aeronautics and Space related to the Technology Committee of the Ministry of Science and Technology during from 1998 to 2000. He was a councilor of Saga University for 2002 and 2003. He also was an executive councilor for the Remote Sensing Society of Japan for 2003 to 2005. He is an Adjunct Professor of University of Arizona, USA since 1998. $\mathrm{He}$ also is Vice Chairman of the Commission-A of ICSU/COSPAR since 2008. He wrote 30 books and published 332 journal papers. 